\title{
A MULTI-INTERSECTION MODEL AND SIGNAL TIMING PLAN ALGORITHM FOR URBAN TRAFFIC SIGNAL CONTROL
}

\author{
Wenbin Hu, Huan Wang, Bo Du, Liping Yan \\ School of Computer, Wuhan University, China \\ Submitted 30 November 2013; resubmitted 25 February 2014; accepted 26 June 2014; \\ first published online 28 August 2014
}

\begin{abstract}
The urban traffic signal control system is complex, non-linear and non-equilibrium in real conditions. The existing methods could not satisfy the requirement of real-time and dynamic control. In order to solve these difficulties and challenges, this paper proposes a novel Multi-Intersection Model (MIM) based on Cellular Automata (CA) and a Multi-Intersection Signal Timing Plan Algorithm (MISTPA), which can reduce the delay time at each intersection and effectively alleviate the traffic pressure on each intersection in the urban traffic network. Our work is divided into several parts: (1) a multi-intersection model based on CA is defined to build the dynamic urban traffic network; (2) MISTPA is proposed, which truly reflects the real-time demand degree to green time of the traffic flow at each intersection. The MISTPA is composed Single Intersection Volume Algorithm (SIVA), Single-Lane Volume Algorithm (SLVA) and single intersection signal timing plan algorithm (SISTPA). Extensive experiments show that when the saturation is greater than 0.3, the MIM and the MISTPA achieve good performance, and can significantly reduce the vehicle delay time at each intersection. The average delay time of the traffic flow at each intersection can obviously be reduced. Finally, a practical case study demonstrates that the proposed model and the corresponding algorithm are correct and effective.
\end{abstract}

Keywords: cellular automata; signal timing plan; delay time; multi-intersection model; urban traffic signal control.

\section{Introduction}

Nowadays many cities are suffering from severe daily urban traffic congestion, which causes the enormous social and economic problems (Triantis et al. 2011). Since changes in the urban area infrastructure are usually not possible, researchers often suppose that an efficient scheduling of traffic signal can help to reduce these problems by improving the flow of vehicles through the cities. Taylor and Heydecker (2014) derives novel approximations for the equilibrium mean and also variance and utilization, using functions linking traffic intensity with green period capacity. With three moments, equilibrium probability distributions can be estimated for which a method based on a doubly nested geometric distribution is described. Christofa et al. (2013) presents a person-based traffic responsive signal control system for transit signal priority on conflicting transit routes. He proposed a mixed-integer nonlinear program is formulated, which minimizes the total person delay at an intersection while assigning priority to the transit vehicles based on their passenger occupancy. Nagatani (2008) studied the dynamical behavior of a shuttle bus moving through a traffic signal. The dynamics of the bus is expressed in terms of the nonlinear maps. The bus dynamics is controlled by varying the loading parameter, the cycle time of signal, and the degree of speedup. The typical urban traffic signal control systems are TRANSYT (Wong et al. 2002), SCOOT (Robertson, Bretherton 1991) and SCATS (Sims, Dobinson 1980). There are several difficulties and challenges in the previous traffic signal optimization research (Wiering et al. 2004; Kerner 2002; McKenney, White 2013):

- many optimization-based methods are based on off-line models, using historical data to optimize traffic signal within the urban road network. However, historical data cannot always accurately reflect the current traffic state (Balaji et al. 2010);

- with an expansion of the urban road network, plenty of traffic signal control methods cannot realize real-time control;

- a wide range of traffic signal optimization research may be unable to reflect the real traffic conditions, for these researches typically assume a very simple traffic infrastructure, such as the traffic network consisting of a few intersections.

Corresponding author: Wenbin $\mathrm{Hu}$

E-mail: hwb@whu.edu.cn 
In this paper, we propose a novel Multi-Intersection Model (MIM) and a Multi-Intersection Signal Timing Plan Algorithm (MISTPA), which can reduce the vehicle delay time at each intersection and effectively alleviate the traffic pressure at each intersection of the urban traffic network. The main contributions of this paper are described as follows:

- the proposed MIM is to map the urban traffic network to a virtual Cellular Automata (CA) model, which completes the construction of MIM;

- the MISTPA is proposed to calculate the current phase's accurate time of the traffic signal at each intersection.

The rest parts of this paper are organized as follows. Section 1 describes the related works of urban traffic model and typical traffic signal optimization approaches. Section 2 presents the problem description and elaborates the construction of the MIM. Section 3 details MISTPA. Section 4 presents a large number of experiments to validate the proposed model and MISTPA. Final section draws the conclusions and perspectives.

\section{Related Works}

Regarding the related papers published in the traffic signal control fields, the research works mainly to adopt the deterministic or stochastic approaches, which had achieved great progress in problem description, modeling and solving algorithm. The merit and demerit of the typical models and algorithms for traffic signal fields are concluded in Table 1.

The signal control optimization methods in Table 1 had their own shortcomings. While in addressing prob- lems with complex, non-linear, non-equilibrium, for example, urban traffic signal control, CA was an effective approach. Any system with many discrete elements could use CA to construct model, as urban traffic signal control system could be divided into many components, and each component followed some simple rules. Systems composed of these components could produce very complex behaviors (Mamei et al. 2005). Therefore, using cellular automata to establish the urban traffic signal control model was greatly significant. CA models could show a large number of traffic scenarios varying from quite simple ones to the extremely complex ones (Spyropoulou 2007), such as the scene of simulation of traffic lane reduction (Nassab et al. 2006), simulation of change behaviors of vehicles by changing lanes ( $\mathrm{Li}$ et al. 2005) and simulation of mixed traffic (Lan, Chang 2003, 2005). In literature (Wei el al. 2005), although the CA has been used to investigate urban traffic for several decades, it is mainly applied in microscopic traffic. In a microscopic traffic model, vehicle velocity was used to represent the state variable. The space was discretized into many cells and the one or more vehicles occupied each cell.

This paper focuses on the urban traffic signal control under the uncertainty. In order to solve the above problems of existing urban traffic signal control systems, this paper proposes a novel macroscopic MIM based on CA and a MISTPA. In our proposed method, multi-intersections within an urban road network are mapped into a virtual MIM model of CA, which realizes the construction of the MIM. After establishing the model of multi-intersections, the proposed MISTPA can decrease vehicle delay time at each intersection.

Table 1. The merits and demerits of the typical models and algorithms for traffic signal fields

\begin{tabular}{|c|c|c|c|}
\hline Classification & Models and algorithms & References & Merit and demerit \\
\hline \multirow{2}{*}{$\begin{array}{l}\text { Deterministic } \\
\text { approaches }\end{array}$} & $\begin{array}{l}\text { Partial differential } \\
\text { equations }\end{array}$ & Soh et al. 2009 & $\begin{array}{l}\text { Difficult to solve, suitable for a particular aspect } \\
\text { of traffic systems }\end{array}$ \\
\hline & $\begin{array}{l}\text { Random point process } \\
\text { theory }\end{array}$ & Baras et al. 1979 & Hard to achieve real-time control \\
\hline \multirow{5}{*}{$\begin{array}{l}\text { Stochastic } \\
\text { approaches }\end{array}$} & Fuzzy control & $\begin{array}{l}\text { Pappis, Mamdani 1977; } \\
\text { Wei et al. 2001; } \\
\text { Niittymäki, Pursula } 2000\end{array}$ & Effective for small traffic network \\
\hline & Neural networks & $\begin{array}{l}\text { Wei 2001; } \\
\text { Wei, Zhang } 2002\end{array}$ & $\begin{array}{l}\text { Had a very simple structure, its neural performance } \\
\text { is susceptible to the traffic volumes, so that they had } \\
\text { to relearn an effective control approach }\end{array}$ \\
\hline & Decision support systems & Almejalli et al. 2007 & $\begin{array}{l}\text { Effectively select the proper strategy, difficult to } \\
\text { obtain and maintain historical data and expert } \\
\text { knowledge }\end{array}$ \\
\hline & $\begin{array}{l}\text { Evolutionary computation } \\
\text { and swarm algorithm }\end{array}$ & $\begin{array}{l}\text { De Oliveira, Bazzan 2006; } \\
\text { García-Nieto et al. 2012; } \\
\text { Montana, Czerwinski } 1996 ; \\
\text { Sanchez-Medina } \text { et al. } 2010\end{array}$ & $\begin{array}{l}\text { Requires a great deal of computing resources } \\
\text { (a number of simulations). The cost of simulations } \\
\text { is high. When traffic network is large enough, } \\
\text { real-time control might be impossible }\end{array}$ \\
\hline & Reinforced learning & $\begin{array}{l}\text { Wiering et al. 2004; } \\
\text { Bazzan et al. } 2010\end{array}$ & $\begin{array}{l}\text { This method was effective, but the performance } \\
\text { of these approaches is sensitive to the change } \\
\text { of traffic state parameters }\end{array}$ \\
\hline
\end{tabular}




\section{Problem Description and MIM}

\subsection{Problem Description}

In this paper, the urban traffic adaptive control theory based on CA is adopted to construct the adaptive control model of urban intelligent traffic system. According to the relationship between the local intersection and its neighbors, this paper presents an ideal that urban traffic control is converted into a problem with two-dimensional CA to construct the adaptive control model. In order to meet the requirements of a real-time control of urban traffic signal, the principle of hierarchical progress is used to drive the evolution of urban traffic signal pattern, which can solve some uncertain problems in urban traffic signal control. Modeling of MIM within an urban traffic road network in this paper has three steps as follows:

- discretizing cells;

- explicit of cells information;

- discretizing cells state.

The urban traffic signal control system is discretized into a two-dimensional grid that regards each intersection as a cell. According to the relationship between the local cell and its neighbors, the urban signal control system can be expressed in a virtual CA model, which is shown in Fig. 1. The adaptive evolution processes of all cells run on the virtual CA model based on an attribute matrix. Evolutionary optimization of cell attribute (traffic flow) combined with signal timing plan strategy calculates green wave time of each phase in each traffic signal to achieve the purpose of easing urban traffic pressure.

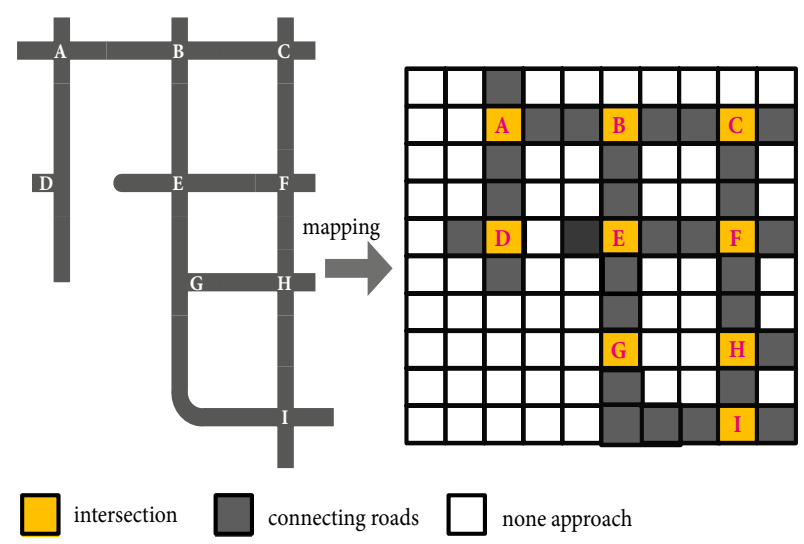

Fig. 1. The mapping between multi-intersections and virtual cell

\subsection{Problem Solving Steps}

The problem solving steps are described as follows:

- obtaining the structure of urban traffic road network, and setting traffic parameters (including the lands number) of each intersection within the traffic road network. The number of intersections within the traffic road network is defined as $N$, and these intersections are marked with the number of 1 to $N$;
- constructing the urban traffic signal adaptive control system based on CA model, including cell, cell state and CA rule (detailed in Section 2.3);

- obtaining the values of parameters, such as the initial number of vehicles in each intersection, the phase state of traffic signal in each intersection, and converting these parameters into traffic density and traffic velocity of each intersection;

- adding the traffic density and traffic velocity to the corresponding cell, and using CA rules to concurrently evolve each cell at the same time step. After several generations evolutions each cell will get the traffic density and traffic velocity;

- converting the parameters of the evolved traffic density and traffic velocity of each cell into the vehicle flow pressure of each cell;

- calculating traffic signal green wave time of current phase in each intersection (algorithm MISTPA, which is detailed in Section 3).

\subsection{MIM based on CA}

\subsubsection{Definitions}

Assumptions and definitions are defined as follows:

- Cycle Length (CL). CL is the total traffic signal time of all phases in the intersection. In this paper, $C L$ is a fixed value;

- Traffic signal control time step $\left(t_{\text {step }}\right) \cdot t_{\text {step }}$ is evolutionary time step of cells, which is set as $5 \mathrm{~s}$ in this paper;

- Evolutionary Generations of the Cell (EGC). EGC is the needed generation times for cell evolution, which usually ranges from 8 to 10 ;

- Phase switching. Considering the reality of the urban traffic situation, this paper adopts four kinds of phases of traffic signal within the urban traffic road network. These phases are shown in Fig. 2. The four kinds of traffic signal phase in cycles alternately change.

\subsubsection{Cell}

In this paper, each intersection within an urban traffic road network is regarded as a cell. The relationship of cells uses the relative position of geographic topology in the urban traffic road network to describe. So a Virtual Adjacency Matrix (VAM) can be used to express the neighborhood relationship of intersections within the urban traffic road network. The VAM is shown in Eq. (1):

$$
\mathrm{VAM}=\left[\begin{array}{l}
b_{11}, b_{12}, b_{13}, b_{14} \\
b_{21}, b_{22}, b_{23}, b_{24} \\
\ldots \ldots \ldots \ldots \ldots \ldots \ldots \\
b_{n 1}, b_{n 2}, b_{n 3}, b_{n 4}
\end{array}\right],
$$

where: $n$ is the number of intersections in the urban traffic road network; $b$ is an intersection in an urban traffic road network, which is regarded as a cell in VAM. For example, $b_{12}$ is an intersection of road 1 and road 2 . 


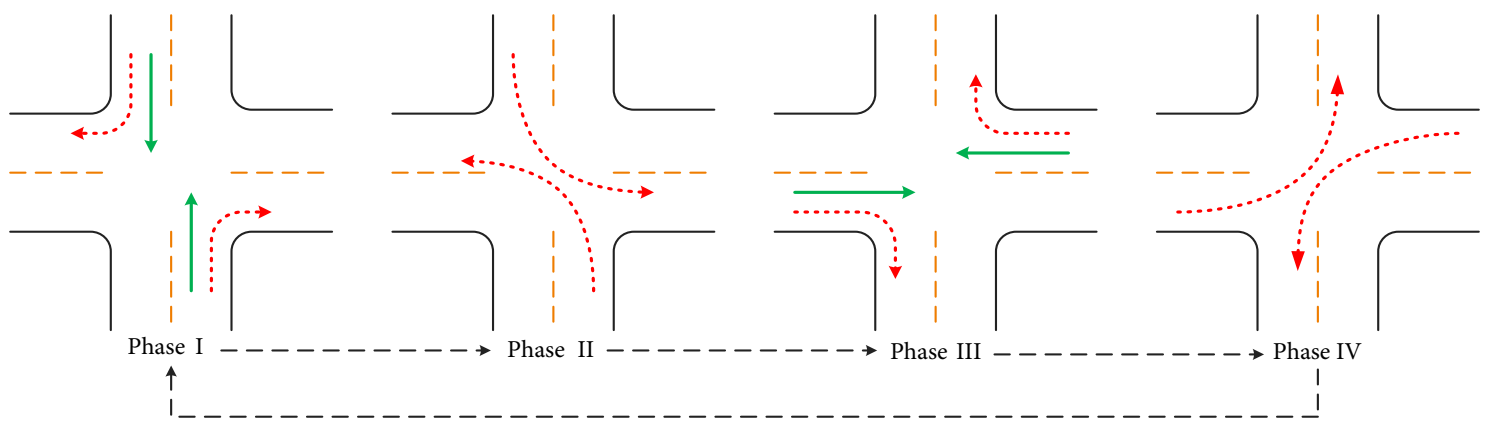

Fig. 2. Four kinds of traffic signal phase

\subsubsection{Cell State}

By analyzing a large number of parameters in the traffic flow information, this paper defines a Vehicle Flow Pressure $(v f p)$ of each road in the intersection as the state of the cell. $v f p$ reflects the demand degree to green light of traffic flow at each road of the intersections, and it is closely related to the queue length at each road of the intersections. $v f p$ is shown in Eq. (2):

$$
v f p=\sum_{k=1}^{K} \text { STotalVolume } e_{k}
$$

where: $K$ represents the number of lanes at road $j$ of intersection $i$; STotalVolume ${ }_{k}$ represents traffic flow of lane $k$ at road $j$ of intersection $i$. The unit of traffic flow is vehicle number per one hour $[\mathrm{veh} / \mathrm{h}]$.

The vector variable $S_{i}$ represents the state of cell, which is shown in Eq. (3) and represents a set $v f p$ of all the roads in the intersection. If the road $j$ of the intersection $i$ does not exist, set $v f p_{j}=-1$.

$$
S_{i}=\left\{v f p_{j} \mid j=1-4\right\} \text {. }
$$

\subsubsection{CA Rule}

The paper adopts Von Neumann neighborhood, and evolutionary rule of CA based on Von Neumann neighborhood, shown in Eq. (4):

$$
S_{i}^{k+1}(t)=F\left(S_{i}^{k}(t), S_{i 1}^{k}(t), S_{i 2}^{k}(t), S_{i 3}^{k}(t), S_{i 4}^{k}(t)\right),
$$

where: $S_{i}^{k+1}$ expresses the state of cell $i$ after evolution of the $k+1$ times generation; $S_{i j}^{k}(j=1,2,3,4)$ represents the state of cells which is related to cell $i ; F$ is nonlinear state updated function; $t$ is the absolute time at the beginning of time step $t_{\text {step }} ; k$ is evolutionary generations, and if $k$ is zero, $S_{i}$ expresses the detected actual traffic data. Evolutionary computation of each intersection within the urban traffic road network is in parallel, and after several generation evolutions, we can get a set of stable and global coordinated $v f p$, which is shown in Fig. 3, where $t_{\text {step }}$ is detailed in Section 2.3.1, and $t_{\text {step }}$ is equally divided into $k$ segments; $t_{i}(i=1,2,3, \cdots, k)$ is a segment time; $G T_{\text {start }}$ is the beginning time of green phase of traffic signal; min $G T$ represents minimum time of each

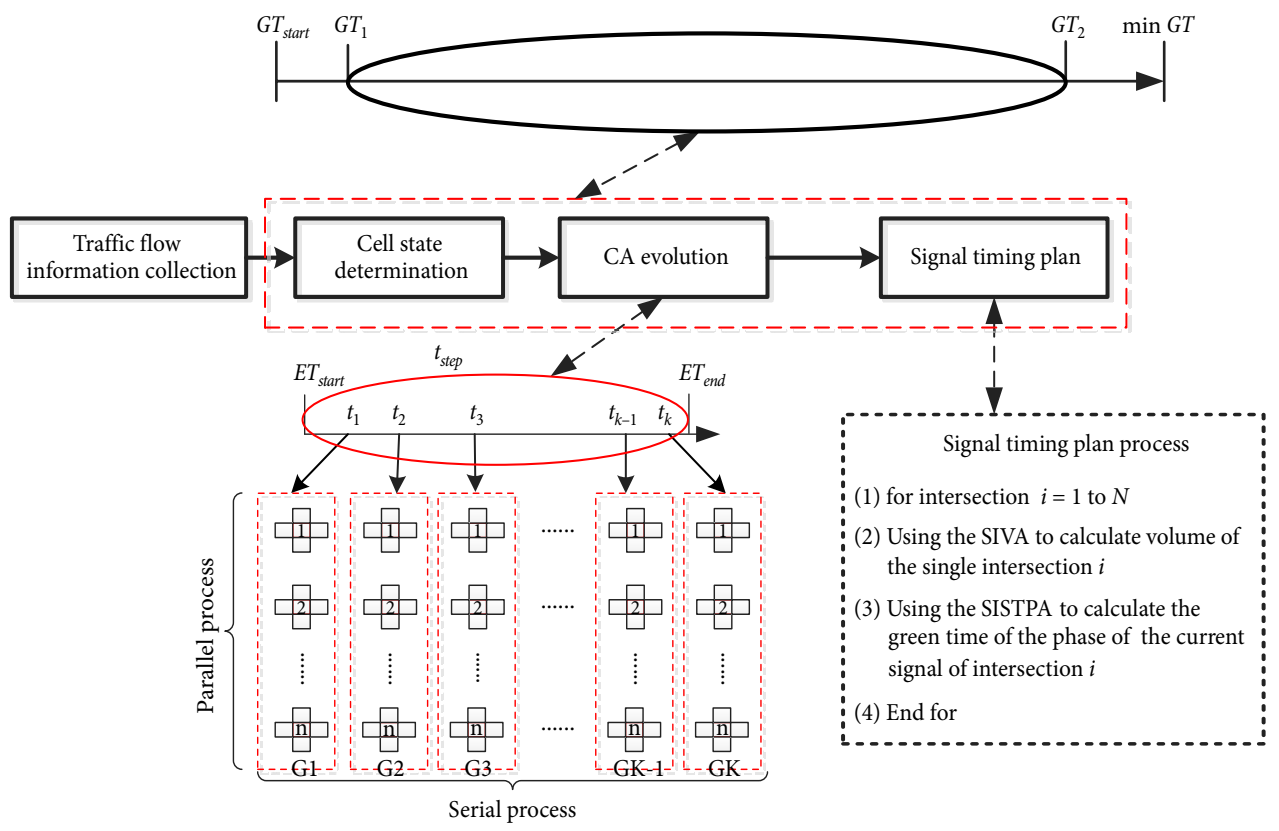

Fig. 3. The process of urban traffic signal adaptive control 
phase of each traffic signal during a cycle; $E T_{\text {start }}$ and $E T_{\text {end }}$ express the beginning and the end evolutionary time of cell state; $G T_{1}$ and $G T_{2}$ denote the beginning and the end of the procedure of cell state determination, CA evolution and signal timing plan; $G_{i}$ represents the $i$ th $(i=1,2,3, L, k)$ generation evolution of cell. During $t_{\text {step }}$, cell collects traffic flow information of intersection, and then converts the information into the state of cell. After several generation evolutions of cell state, the evolutionary result provides the necessary decision support for signal timing plan of the current phase.

There are some similarities between the ordinary fluid and the urban traffic flow (Pipes 1953), so this paper introduces Navier-Stokes equation of the ordinary fluid into the urban traffic flow. The Navier-Stokes equation is shown in Eq. (5):

$$
\rho \cdot \frac{d u_{i}}{d t}=\rho \cdot f_{i}-\frac{\partial p}{\partial x_{i}}+\frac{\partial}{\partial x_{j}}\left(\mu \cdot\left(\frac{\partial u_{i}}{\partial x_{j}}+\frac{\partial u_{j}}{\partial x_{i}}\right)\right),
$$

where: $\rho$ is the fluid density; $f_{i}$ represents the mass force; $u_{i}$ and $u_{j}$ denote $i$ direction and $j$ direction of the fluid velocity; $p$ expresses the intensity of pressure. By mathematical reconstruction of the Eq. (5), it can get the simplified Navier-Stokes equation that can be applied to the urban traffic flow. The simplified Navier-Stokes equation is shown in Eq. (6):

$$
\frac{d u}{d t}=-\frac{1}{\rho} \cdot \nabla p+\tilde{\mu} \cdot \nabla^{2} u,
$$

where: $u$ is the traffic velocity $[\mathrm{m} / \mathrm{s}$ or $\mathrm{km} / \mathrm{h}] ; \nabla p$ represents the pressure gradient force; $\tilde{\mu}$ expresses the viscosity coefficient; $\rho$ denotes the traffic density; $t$ is the time.

Using the finite difference method to decompose the Equation (6) (Kita, Toyoda 2000), we can get the local evolution of CA rule, which is shown in Eq. (7):

$u_{j, k}^{n+1}=\left(1-2 \cdot \lambda_{1}\right) \cdot u_{j, k}^{n}+\lambda_{1} \cdot u_{j+1, k}^{n}+\lambda_{1} \cdot u_{j-1, k}^{n}+\lambda_{2} \cdot \tilde{p},(7)$ where:

$$
\begin{aligned}
& \lambda_{1}=\frac{\tilde{\mu}}{(\Delta x)^{2}} \cdot \Delta t ; \\
& \lambda_{2}=\Delta t ; \\
& \tilde{p}=-\frac{1}{\rho} \cdot \nabla p .
\end{aligned}
$$

\section{MISTPA}

\subsection{Step Workflow of MISTPA}

MISTPA is composed of a Single Intersection Volume Algorithm (SIVA) and a Single Intersection Single Timing Plan Algorithm (SISTPA). The average vehicle volume of intersection $i$ at current signal phase and the total average vehicle volume of interaction $i$ are computed by algorithm SIVA. TotalVolume $i$ represents the total average vehicle volume of interaction $i$; volumeofsignal $_{i}$ represents the average vehicle volume of intersection $i$. The signal green time of intersection $i$ at current signal phase is the ratio of volumeofsignal ${ }_{i}$ and TotalVolume $_{i}$. The steps of MISTPA are detailed in Algorithm MISTPA:

\begin{tabular}{|l|}
\hline Algorithm MISTPA \\
\hline Input: the vehicle flow pressure of each intersection at current \\
signal phase (rules are detailed in Section 2.3.4) \\
Output: the signal green time of each intersection \\
1: FOR $i=1$ to $N$ \\
2: Computing volumeofsignal ${ }_{i}$ and TotalVolume ${ }_{i}$ by SIVA \\
(SIVA is detailed in Section 3.2) \\
3: Computing signal green time of each intersection at current \\
signal phase by SISTPA (SISTPA is detailed in Section 3.3) \\
4: END FOR
\end{tabular}

\subsection{SIVA}

SIVA is composed by two parts:

- computing volumeofsignal $i$;

- computing TotalVolume . $_{\text {. }}$

The symbols in SIVA are defined in Table 2. The steps of SIVA are detailed in Algorithm SIVA.

\subsection{SISTPA}

The volumeofsignal ${ }_{i}$ and TotalVolume ${ }_{i}$ of each intersection are computed by algorithm SIVA. The signal green time Gree $T_{i}$ of each intersection is computed by Eq. (8):

$$
\text { Gree }_{i}=\frac{C L \cdot \text { volumeofsignal }_{i}}{\text { TotalVolume }_{i}} .
$$

The symbols of algorithm SISTPA are defined in Table 3. If Gree $T_{i}$ is smaller than MinT, set MinT= $G r e e T_{i}$, and if $G r e e T_{i}$ is bigger than MaxT, set MaxT= $G r e e T_{i}$, otherwise $G r e e T_{i}$ remains unchanged. The steps of SISTPA are detailed in Algorithm SISTPA.

\section{Performance Evaluation and Comparison}

This section is devoted to the performance evaluation of the proposed model MIM and the algorithm MISTPA. The adopted experiments data and parameters setting will be described in Section 4.1. Section 4.2 details the comparison results of performance of the proposed algorithm under different saturations. Section 4.3 presents a real case study in City of Wuhan, China.

\subsection{Experiment Setup}

In the traffic simulation system, the constraints/conditions are defined as follows (the parameters setting is shown in Table 4):

- the local traffic network of the experiment is a $5 \times 5$ structure, which is shown in Fig. 4 . The intersections are all crossroads and are all numbered from left to right, from up to down in turn. The lanes and lengths of the roads are set as the real conditions;

- the local traffic network is an open region. Traffic flow enters the local traffic network from the boundaries, so the traffic capacity of horizontal and vertical roads should be set. In this experiment, the traffic capacity of horizontal roads is $2000 \mathrm{veh} / \mathrm{h}$, and the traffic capacity of vertical roads is $1400 \mathrm{veh} / \mathrm{h}$; 
Table 2. Symbol definition of SIVA

\begin{tabular}{|l|l|}
\hline \multicolumn{1}{|c|}{ Symbols } & \multicolumn{1}{c|}{ Descriptions } \\
\hline$i$ & Intersection (cell number) \\
\hline$k$ & The road number not in passing state of intersection $i$ at current signal phase \\
edge_count ${ }_{k}$ & The average volume of road $k$ of intersection $i$ at current signal phase \\
num_lanes & Lanes number of road \\
$M_{k}$ & The actual lanes of road $k$ of intersection $i$ at current signal phase \\
avg_cars $k$ & The average volume of road $k$ of intersection $i$ at current signal phase \\
distance 1 & The distance of road 1 in passing state of intersection $i$ at current signal phase \\
distance 2 & The distance of road2 in passing state of intersection $i$ at current signal phase \\
cars_num 1 & The variable of vehicle \\
cars_num 2 & The variable of vehicle \\
$\beta_{s}$ & The vehicle going straight ratio of connecting roads in intersection \\
$\beta_{\text {left }}$ & The vehicle going left ratio of connecting roads in intersection \\
$\beta_{\text {right }}$ & The vehicle going right ratio of connecting roads in intersection \\
\hline
\end{tabular}

Algorithm SIVA

Input: the vehicle flow pressure value of cell $\mathrm{i}$, which is two-way, and is defined as $v f p_{i} 1$ and $v f p_{i} 2$

Output: volumeofsignal $_{i}$ and TotalVolume ${ }_{i}$

1: Initializing the values of volumeofsignal $_{i}$ and TotalVolume ${ }_{i}$ as zero

2: FOR $k=1$ to 2

3: Initializing the values of edge_count ${ }_{k}$ and volumeofsignal $l_{k}$ as zero

4: FOR num_lanes $=1$ to $M_{k}$

5: TotalVolume ${ }_{k}$ is the summation of the vehicle number of lane num_lanes and TotalVolume $k_{k}$

6: volumeofsignal $_{k}=\frac{\text { TotalVolume }_{k}}{M_{k}}$ (details in a Single-Lane Volume Algorithm - SLVA $)$

7: edge_count $_{k}=\frac{\text { volumeofsignal }_{k}+\text { edge_count }}{k}$
8: END FOR

9: TotalVolume $_{i}=$ edge_count $_{k}+$ TotalVolume $_{i}$

10: cars_num $1=v f p_{i} 1+$ distance 1

11: cars_num $2=v f p_{i} 2+$ distance 2

12: END FOR

13: IF the current signal phase of intersection $i$ is phase I or phase III

14: cars_num $1=\frac{\text { cars_num } 1 \cdot\left(\beta_{s}+\beta_{\text {right }}\right)}{\text { lane_phase } 1}$, where: $\beta_{s}$ and $\beta_{\text {right }}$ are the ratio of road 1 ; lane_phase 1 is the total lane number of going straight lane and going right lane in road 1

15: cars_num $2=\frac{\text { cars_num } 2 \cdot\left(\beta_{s}+\beta_{\text {right }}\right)}{\text { lane_phase } 2}$, where: $\beta_{s}$ and $\beta_{\text {right }}$ are the ratio of road2; lane_phase 2 is the total lane number of going straight lane and going right lane in road 2

16: END IF

17: IF the current signal phase of intersection $\mathrm{i}$ is phase II or phase IV 18: cars_num $1=\frac{\text { cars_num } 1 \cdot \beta_{\text {left }}}{\text { lane_phase } 3}$, where: $\beta_{\text {left }}$ is the ratio of road 1 ; lane_phase 3 is the total lane number of going left lane
in 1 . 19: cars_num $2=\frac{\text { cars_num } 2 \cdot \beta_{\text {left }}}{\text { lane_phase } 4}$, where: $\beta_{\text {left }}$ is the ratio of road2; lane_phase4 is the total lane number of going left lane
in road 1

20: END IF

20: volumeofsignal $_{i}=$ cars_num $1+$ cars_num 2

21: TotalVolume $_{i}=$ TotalVolume $_{i}+$ volumeofsignal $_{i}$

Algorithm SLVA

Input: the vehicle volume of each lane, the weight of the vehicle volume. The Observed Frequency is defined as $O F$

Output: the vehicle volume of single-lane

1: Initializing the value of vehicle volume of single-lane as zero

2: FOR $i=1$ to $O F$

3: The value of vehicle volume of single-lane $=$ the value of vehicle volume of single-lane + the product of the $i$ th observed vehicle volume and the weight of the ith observed data

4: END FOR 
Table 3. Symbol definition of SISTPA

\begin{tabular}{|l|l|}
\hline \multicolumn{1}{|c|}{ Symbols } & \multicolumn{1}{c|}{ Descriptions } \\
\hline proportion & The ratio of volumeofsignal ${ }_{i}$ and TotalVolume \\
MinT & The minimum signal green time (constant) \\
MaxT & The maximum signal green time (constant) \\
Offsettime & The offset value of signal green time \\
\hline
\end{tabular}

Algorithm SISTPA

Input: volumeofsignal ${ }_{i}$ and TotalVolume of $_{i}$ each intersection Output: the value of Gree $T_{i}$

1: propotrion $_{i}=\frac{\text { volumeofsignal }_{i}}{\text { TotalVolume }_{i}}$

2: Gree $_{i}=\frac{C L \cdot \text { volumeofsignal }_{i}}{\text { TotalVolume }_{i}}+$ Offsettime

3: IF Gree $T_{i}<$ MinT

4: $M i n T=$ Gree $T_{i}$

5: END IF

6: IF Gree $_{i}>$ MaxT

7: MaxT $=$ Gree $_{i}$

8: END IF

9: Return the value of $G r e e T_{i}$

Table 4. The parameters setting in the experiment

\begin{tabular}{|l|c|c|c|}
\hline \multicolumn{1}{|c|}{ Parameters } & Distribution & Default & Variable \\
\hline Detecting distance & - & - & $\mathrm{Y}$ \\
\hline Simulation step & - & 100 & $\mathrm{Y}$ \\
\hline$t_{\text {step }}$ & - & $5 \mathrm{~s}$ & $\mathrm{~N}$ \\
\hline MinGT & - & $15 \mathrm{~s}$ & $\mathrm{Y}$ \\
\hline MaxGT & - & $60 \mathrm{~s}$ & $\mathrm{Y}$ \\
\hline CL & - & $120 \mathrm{~s}$ & $\mathrm{Y}$ \\
\hline Vehicle length & - & $4 \mathrm{~m}$ & $\mathrm{Y}$ \\
\hline The evolution algebra & - & 10 & $\mathrm{Y}$ \\
\hline$\beta_{\text {left }}$ & - & - & $\mathrm{Y}$ \\
\hline$\beta_{s}$ & - & - & $\mathrm{Y}$ \\
\hline$\beta_{\text {right }}$ & - & - & $\mathrm{Y}$ \\
\hline Vehicle density & - & - & $\mathrm{Y}$ \\
\hline Vehicle speed & - & - & $\mathrm{Y}$ \\
\hline Vehicle arrival rate & Poisson distribution & - & $\mathrm{Y}$ \\
\hline Delay time & - & - & $\mathrm{Y}$ \\
\hline Average delay time & - & - & $\mathrm{Y}$ \\
\hline Vehicle number & Random distribution & - & $\mathrm{Y}$ \\
\hline Vehicle saturation & - & - & $\mathrm{Y}$ \\
\hline
\end{tabular}

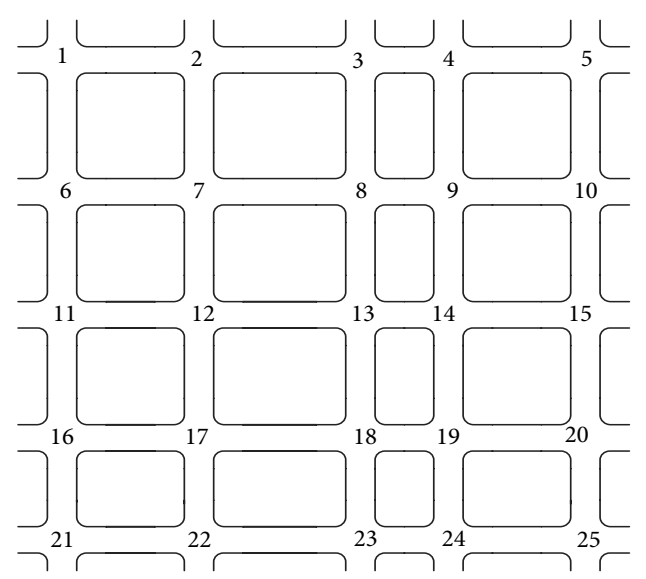

Fig. 4. Local traffic network structures
- the vehicle arrival rate of the boundary intersection admits a Poisson distribution;

- the initialized conditions of experiments under different saturations are the same;

- three strategies are introduced to the experiment to compare the delay time of vehicles. Three strategies are detailed in Table 5.

Table 5. The description of three strategies

\begin{tabular}{|l|l|l|}
\hline \multicolumn{1}{|c|}{ Strategy name } & Abbreviation & \multicolumn{1}{c|}{ Comments } \\
\hline $\begin{array}{l}\text { Signal timing } \\
\text { strategy based } \\
\text { on CA model }\end{array}$ & STSBNCAM & $\begin{array}{l}\text { Using CA model to map } \\
\text { the real road network } \\
\text { and using signal time } \\
\text { planning algorithm }\end{array}$ \\
\hline $\begin{array}{l}\text { Signal timing } \\
\text { strategy without } \\
\text { CA model }\end{array}$ & STSWCAM & $\begin{array}{l}\text { Only using signal time } \\
\text { planning algorithm }\end{array}$ \\
\hline $\begin{array}{l}\text { Signal timing } \\
\text { strategy based } \\
\text { on the fixed } \\
\text { green time }\end{array}$ & STSBFGWT & $\begin{array}{l}\text { Without CA model } \\
\text { and signal time } \\
\text { planning algorithm }\end{array}$ \\
\hline
\end{tabular}

\subsection{Experimental Results and Analysis}

\subsubsection{Simulation under Different Signal Timing Plan Strategies}

The delay time is introduced to be an evolution index of the experiment. The experiments are conducted under different saturations by three different signal time planning strategies in Table 5. The experiment parameters are set as Table 4 . Saturation is the rate of road or intersection traffic flows and traffic capacity of road or intersection, which is normalized as from 0 to 1 . The saturations of this experiment are set ranging from 0.1 to 1 . Fig. 5 is the performance of the average delay time under different saturations. On the basis of these results in Fig. 5, we can draw the following conclusions:

- when the saturation is small (less than 0.3), the three strategies have the similar performance on vehicle delay time because the neighbor intersections have less effect on the local intersection;

- when the saturation is moderate (0.5), SISBNCAM presents a better performance than STSWCAM and STSBFGWT. SISBNCAM can dramatically reduce the average delay time;

- when the saturation is big (more than 0.7), SISBNCAM has a better performance than STSWCAM and STSBFGWT. SISBNCAM optimizes the green time of current phase by prediction and can dramatically reduce the average delay time.

\subsubsection{Comparison Experiment}

We compare the proposed algorithm with three other state-of-the-art algorithms mentioned in Section 1, including SIA-PSO (Swarm Intelligent Approach-Particle Swarm Optimization) (García-Nieto et al. 2012), SOCA (Self-Organizing Control Approach) (Wei et al. 2005) and GA+CC (Genetic Algorithm and Cluster Computing) (Sanchez-Medina et al. 2010). 
a)
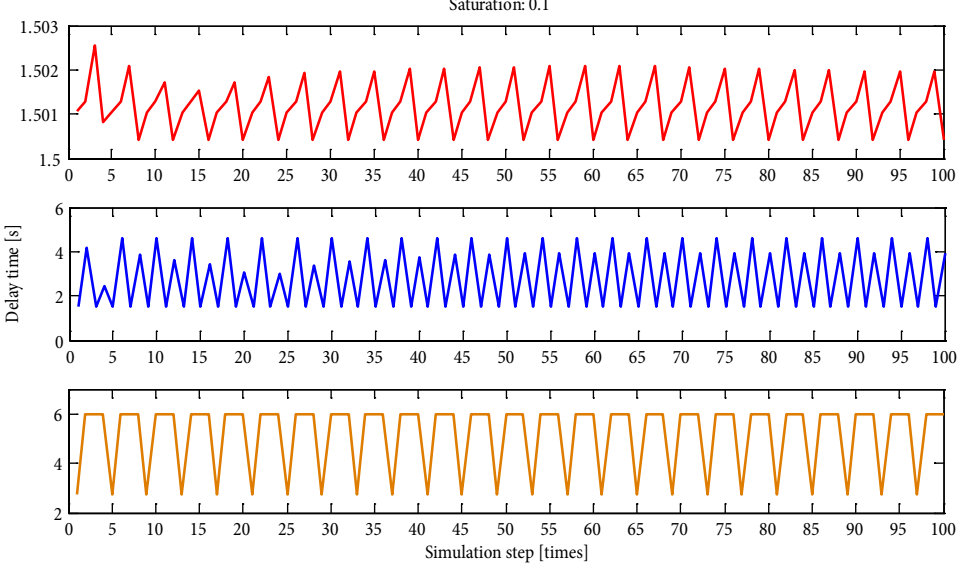

b)

Saturation: 0.3
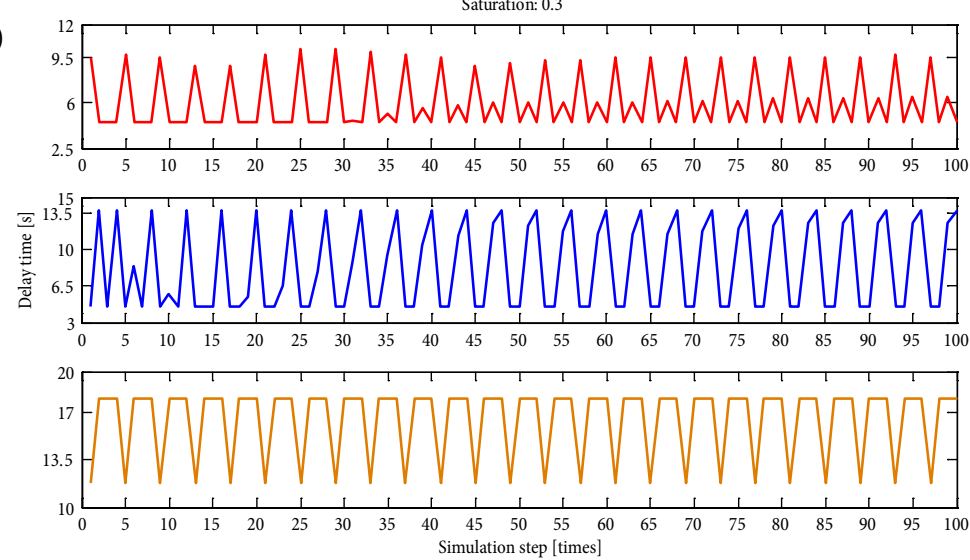

c)
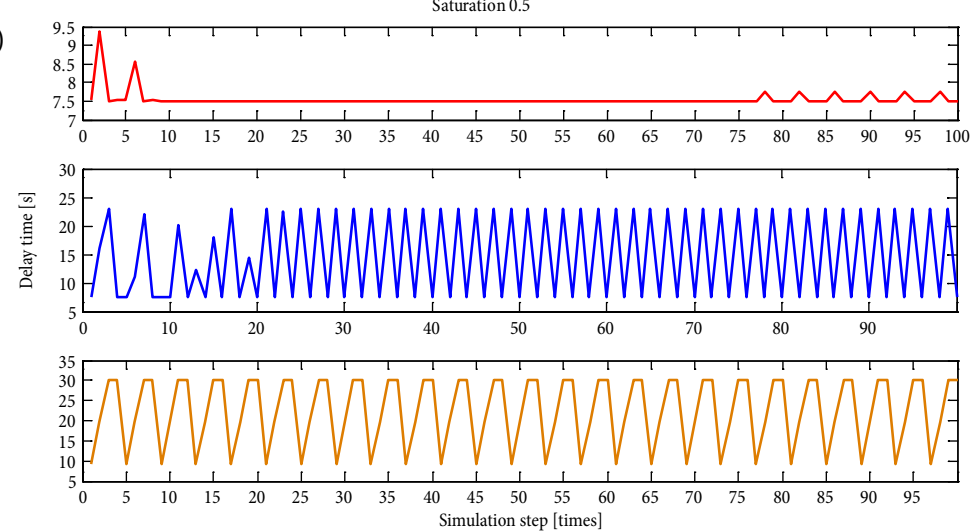

d)
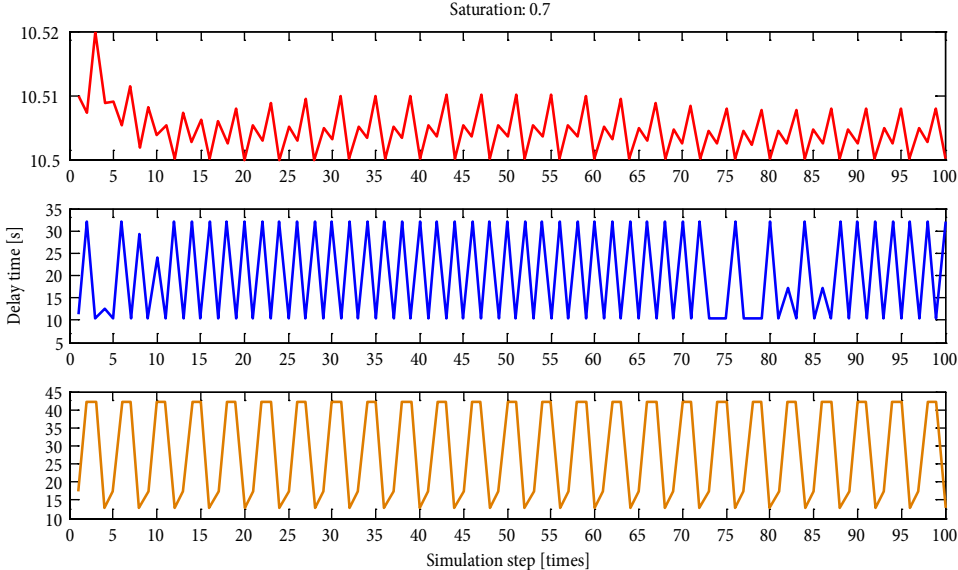
e)
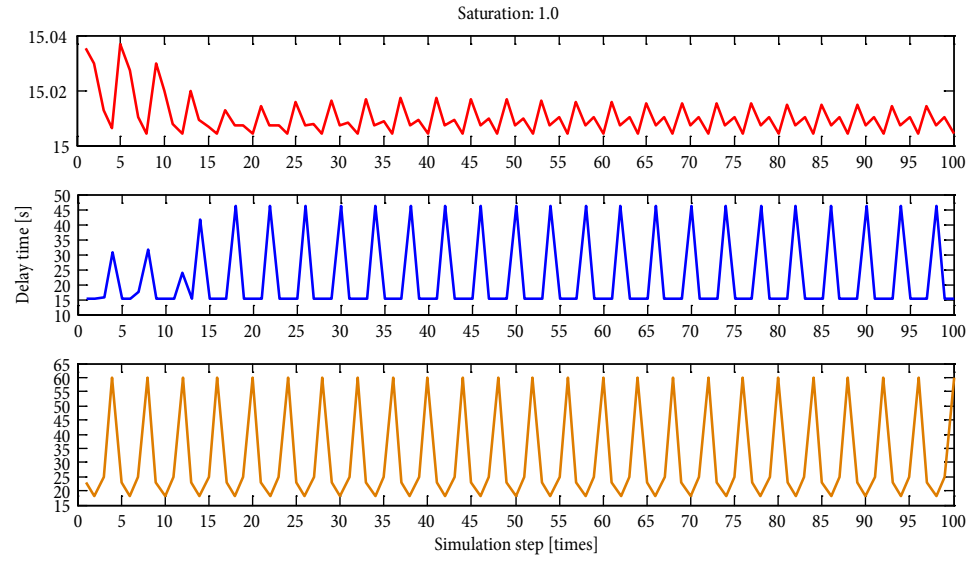

- STSBNCAM STSWCAM $\longrightarrow$ STSBNFGWT

Fig. 5. The average delay time under different saturations: $\mathrm{a}$ - saturation $=0.1 ; \mathrm{b}-$ saturation $=0.3$; $\mathrm{c}-$ saturation $=0.5 ; \mathrm{d}-$ saturation $=0.7 ; \mathrm{e}-$ saturation $=1.0$

These three algorithms mentioned above are the most representative ones of the swarm intelligent approach, reinforced learning and CA approach and the hybrid approach described in Section 1. In the comparison experiments, we implement these algorithms since the corresponding references detail the algorithm steps.

The performance of MISTPA and other state-ofthe-art algorithms are given in Table 6. Based on these results of Table 6, we can draw the following conclusions on the performance on average delay time:

- it is apparent from the results that MISTPA has better performance than SIA-PSO, SOCA and GA+CC. In most cases, MISTPA provides a lower average vehicle delay time than other state-ofthe-art algorithms;

- with the increment of saturation, the advantage of MISTPA is more obvious, which shows that MISTPA is more efficient than other state-of-theart algorithms in solving problems under bigger saturation conditions.

\subsection{Case Study}

A real local traffic network of Hongshan District, Wuhan City, China, is shown in Fig. 6. There is no road name in Fig. 6 in order that the relation between intersections can be distinctly identified. In this real case study, the following constraints/conditions are defined. The constraints/conditions and parameters are set according to Table 4 in Section 4.1.

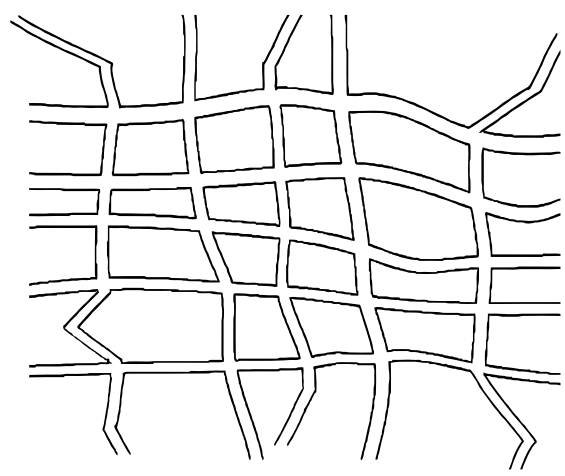

Fig. 6. Local traffic networks

We simulate the traffic system under the different conditions (from under saturated to saturated). The average delay time and results from a set of simulation experiments are shown in Table 7. Based on the simulation results, we can draw the following conclusions:

- The simulation results show that the STSBNCAM provides a lower average vehicle delay time than STSWCAM and STSBFGWT;

- In addition, Table 7 shows that the effects of the three strategies are almost identical in low saturation, because the neighbors' flow pressures almost have no effect on the local intersection when the traffic demands are low. With the increment of saturation, STSBNCAM is obviously superior. When traffic demand is large, through

Table 6. Simulation data and comparison with other state-of-the-art algorithms

\begin{tabular}{|c|c|c|c|c|c|}
\hline Cases & Saturation & $\begin{array}{c}\text { MISTPA } \\
\text { Average delay time [s] }\end{array}$ & $\begin{array}{c}\text { SIA-PSO } \\
\text { Average delay time [s] }\end{array}$ & $\begin{array}{c}\text { SOCA } \\
\text { Average delay time [s] }\end{array}$ & $\begin{array}{c}\text { GA+CC } \\
\text { Average delay time [s] }\end{array}$ \\
\hline 1 & 0.1 & 1.487 & 1.522 & 1.432 & 1.421 \\
\hline 2 & 0.3 & 6.091 & 6.871 & 6.111 & 6.132 \\
\hline 3 & 0.5 & 7.432 & 7.912 & 7.621 & 7.591 \\
\hline 4 & 0.7 & 10.499 & 11.233 & 10.985 & 10.796 \\
\hline 5 & 1.0 & 15.129 & 17.912 & 15.654 & 15.642 \\
\hline
\end{tabular}


the perception to neighbors' states, the signals can be coordinated in traffic network to reduce the unnecessary delay time of forthcoming flow at a leisure intersection. Thus, from the simulative results, the STSBNCAM based on the proposed model and algorithm is effective in urban traffic signal planning.

Table 7. Simulation data and comparisons

\begin{tabular}{|c|c|c|c|c|}
\hline \multirow{2}{*}{ Cases } & \multirow{2}{*}{ Saturation } & \multicolumn{4}{|c|}{ Average delay time [s] } \\
\cline { 3 - 5 } & & STSBNCAM & STSWCAM & STSBFGWT \\
\hline 1 & 0.1 & 1.502 & 2.312 & 5.184 \\
\hline 2 & 0.3 & 5.938 & 8.238 & 16.445 \\
\hline 3 & 0.5 & 7.516 & 14.213 & 22.172 \\
\hline 4 & 0.7 & 10.515 & 19.771 & 28.507 \\
\hline 5 & 1.0 & 15.012 & 27.413 & 31.430 \\
\hline
\end{tabular}

\section{Conclusions and Future Work}

Urban traffic signal planning is a complex real control problem; the main difficulty includes the modeling complication and difficulty in real controlling. This paper proposes a multi-intersection model based on CA and a novel multi-intersection signal timing plan algorithm to solve the problem.

Extensive experiments are conducted to evaluate the proposed model and corresponding algorithm, which show that they can dramatically reduce the vehicle delay time and ease the pressure of urban traffic network:

- MISTPA can compute the green time of current signal phase according to the real time traffic flow, which reduces the delay time of vehicle in each intersection;

- STSBNCAM can predict the neighbors' states by CA evolution rules and can use MISTPA to compute the green time. Because of the discreteness and parallelism of CA, the real-time processing of traffic signal planning can be assured;

- the real case study shows that STSBNCAM provides a lower average vehicle delay time than the other strategies, which proves that the proposed model and algorithm are efficient and can direct practical application well.

Despite the above progress in our proposed method, further research is still needed. Because the MISTPA takes traffic flow as the research object, it does not consider the factors of microcosmic traffic. So how to express the behaviors of microcosmic traffic is the main research direction of MISTPA, which can extend the application of MISTPA and satisfy the real condition of urban traffic signal planning.

\section{Acknowledgements}

This work is partially supported by National Natural Science Foundation, China (No.70901060); Hubei Province Natural Science Foundation (No. 2011CDB461).

\section{References}

Almejalli, K.; Dahal, K.; Hossain, M. A. 2007. Intelligent traffic control decision support system, Lecture Notes in Computer Science 4448: 688-701.

http://dx.doi.org/10.1007/978-3-540-71805-5_75

Balaji, P. G.; German, X.; Srinivasan, D. 2010. Urban traffic signal control using reinforcement learning agents, IET Intelligent Transport Systems 4(3): 177-188.

http://dx.doi.org/10.1049/iet-its.2009.0096

Baras, J. S.; Levine, W. S.; Lin, T. 1979. Discrete-time point processes in urban traffic queue estimation, IEEE Transactions on Automatic Control 24(1): 12-27.

http://dx.doi.org/10.1109/TAC.1979.1101944

Bazzan, A. L. C.; De Oliveira, D.; Da Silva, B. C. 2010. Learning in groups of traffic signals, Engineering Applications of Artificial Intelligence 23(4): 560-568.

http://dx.doi.org/10.1016/j.engappai.2009.11.009

Christofa, E.; Papamichail, I.; Skabardonis, A. 2013. Personbased traffic responsive signal control optimization, IEEE Transactions on Intelligent Transportation Systems 14(3): 1278-1289. http://dx.doi.org/10.1109/TITS.2013.2259623

De Oliveira, D.; Bazzan, A. L. C. 2006. Traffic lights control with adaptive group formation based on swarm intelligence, Lecture Notes in Computer Science 4150: 520-521. http://dx.doi.org/10.1007/11839088_61

García-Nieto, J.; Alba, E.; Carolina Olivera, A. 2012. Swarm intelligence for traffic light scheduling: application to real urban areas, Engineering Applications of Artificial Intelligence 25(2): 274-283.

http://dx.doi.org/10.1016/j.engappai.2011.04.011

Kerner, B. S. 2002. Empirical macroscopic features of spatialtemporal traffic patterns at highway bottlenecks, Physical Review E 65. http://dx.doi.org/10.1103/PhysRevE.65.046138

Kita, E.; Toyoda, T. 2000. Structural design using cellular automata, Structural and Multidisciplinary Optimization 19(1): 64-73. http://dx.doi.org/10.1007/s001580050086

Lan, L. W.; Chang, C.-W. 2005. Inhomogeneous cellular automata modeling for mixed traffic with cars and motorcycles, Journal of Advanced Transportation 39(3): 323-349. http://dx.doi.org/10.1002/atr.5670390307

Lan, L. W.; Chang, C.-W. 2003. Motorbike’s moving behavior in mixed traffic: particle-hopping model with cellular automata, Journal of the Eastern Asia Society for Transportation Studies 5: 23-37.

Li, K.-P.; Gao, Z.-Y.; Ning, B. 2005. Cellular automaton model for railway traffic, Journal of Computational Physics 209(1): 179-192. http://dx.doi.org/10.1016/j.jcp.2005.03.016

Mamei, M.; Roli, A.; Zambonelli, F. 2005. Emergence and control of macro-spatial structures in perturbed cellular automata, and implications for pervasive computing systems, IEEE Transactions on Systems, Man and Cybernetics, Part A: Systems and Humans 35(3): 337-348. http://dx.doi.org/10.1109/TSMCA.2005.846379

McKenney, D.; White, T. 2013. Distributed and adaptive traffic signal control within a realistic traffic simulation, Engineering Applications of Artificial Intelligence 26(1): 574-583. http://dx.doi.org/10.1016/j.engappai.2012.04.008

Montana, D. J.; Czerwinski, S. 1996. Evolving control laws for a network of traffic signals, in Genetic Programming 1996: Proceedings of the First Annual Conference, 28-31 July 1996. Stanford University, 333-338.

Nagatani, T. 2008. Dynamics and schedule of shuttle bus controlled by traffic signal, Physica A: Statistical Mechanics and its Applications 387(23): 5892-5900.

http://dx.doi.org/10.1016/j.physa.2008.06.038 
Nassab, K.; Schreckenberg, M.; Boulmakoul, A.; Ouaskit, S. 2006. Effect of the lane reduction in the cellular automata models applied to the two-lane traffic, Physica A: Statistical Mechanics and its Applications 369(2): 841-852. http://dx.doi.org/10.1016/j.physa.2006.01.073

Niittymäki, J.; Pursula, M. 2000. Signal control using fuzzy logic, Fuzzy Sets and Systems 116(1): 11-22. http://dx.doi.org/10.1016/S0165-0114(99)00034-2

Pappis, C. P.; Mamdani, E. H. 1977. A fuzzy logic controller for a traffic junction, IEEE Transactions on Systems, Man and Cybernetics 7(10): 707-717. http://dx.doi.org/10.1109/TSMC.1977.4309605

Pipes, L. A. 1953. An operational analysis of traffic dynamics, Journal of Applied Physics 24(3): 274-281. http://dx.doi.org/10.1063/1.1721265

Robertson, D. I.; Bretherton, R. D. 1991. Optimizing networks of traffic signals in real time-the SCOOT method, IEEE Transactions on Vehicular Technology 40(1): 11-15. http://dx.doi.org/10.1109/25.69966

Sanchez-Medina, J. J.; Galan-Moreno, M. J.; Rubio-Royo, E. 2010. Traffic signal optimization in "La Almozara" district in Saragossa under congestion conditions, using genetic algorithms, traffic microsimulation, and cluster computing, IEEE Transactions on Intelligent Transportation Systems 11(1): 132-141. http://dx.doi.org/10.1109/TITS.2009.2034383

Sims, A. G.; Dobinson, K. W. 1980. The Sydney coordinated adaptive traffic (SCAT) system philosophy and benefits, IEEE Transactions on Vehicular Technology 29(2): 130-137. http://dx.doi.org/10.1109/T-VT.1980.23833

Soh, A. C.; Khalid, M.; Marhaban, M. H.; Yusof, R. 2009. Modeling of a multilane-multiple intersection based on queue theory and standard approach techniques, Simulation Modelling Practice and Theory 17(6): 1081-1105. http://dx.doi.org/10.1016/j.simpat.2009.03.008

Spyropoulou, I. 2007. Modelling a signal controlled traffic stream using cellular automata, Transportation Research Part C: Emerging Technologies 15(3): 175-190. http://dx.doi.org/10.1016/j.trc.2007.04.001

Taylor, N. B.; Heydecker, B. G. 2014. The effect of green time on stochastic queues at traffic signals, Transportation Planning and Technology 37(1): 3-19. http://dx.doi.org/10.1080/03081060.2013.844907

Triantis, K.; Sarangi, S.; Teodorović, D.; Razzolini, L. 2011. Traffic congestion mitigation: combining engineering and economic perspectives, Transportation Planning and Technology 34(7): 637-645.

http://dx.doi.org/10.1080/03081060.2011.602845

Wei, C.-H. 2001. Analysis of artificial neural network models for freeway ramp metering control, Artificial Intelligence in Engineering 15(3): 241-252. http://dx.doi.org/10.1016/S0954-1810(01)00019-X

Wei, J.; Wang, A.; Du, N. 2005. Study of self-organizing control of traffic signals in an urban network based on cellular automata, IEEE Transactions on Vehicular Technology 54(2): 744-748. http://dx.doi.org/10.1109/TVT.2004.841536

Wei, H.; Yong, W.; Xuanqin, M.; Yan, W. 2001. A cooperative fuzzy control method for traffic lights, in 2001 IEEE Intelligent Transportation Systems Proceedings, 25-29 August 2001, Oakland, CA, USA, 185-188.

http://dx.doi.org/10.1109/ITSC.2001.948653

Wei, W.; Zhang, Y. 2002. FL-FN based traffic signal control, in Proceedings of the 2002 IEEE International Conference on Fuzzy Systems, 2002: FUZZ-IEEE'02, 12-17 May 2002, Honolulu, HI, US, 1: 296-300.

http://dx.doi.org/10.1109/FUZZ.2002.1005004
Wiering, M.; Van Veenen, J.; Vreeken, J.; Koopman, A. 2004. Intelligent Traffic Light Control. Technical Report UUCS-2004-029. Institute of Information and Computing Sciences, Utrecht University, Netherlands. 30 p. Available from Internet: http://www.cs.uu.nl/research/techreps/repo/ CS-2004/2004-029.pdf

Wiering, M.; Vreeken, J.; Van Veenen, J.; Koopman, A. 2004. Simulation and optimization of traffic in a city, in Proceedings of the 2004 IEEE Intelligent Vehicles Symposium, 14-17 June 2004, Parma, Italy, 453-458. http://dx.doi.org/10.1109/IVS.2004.1336426

Wong, S. C.; Wong, W. T.; Leung, C. M.; Tong, C. O. 2002. Group-based optimization of a time-dependent TRANSYT traffic model for area traffic control, Transportation Research Part B: Methodological 36(4): 291-312. http://dx.doi.org/10.1016/S0191-2615(01)00004-2 